\title{
Curative resection of leiomyosarcoma with resection and reconstruction of inferior vena cava
}

\author{
Yashiro Motooka ${ }^{1}$, Daisuke Hashimoto ${ }^{1}$, Hisashi Sakaguchi ${ }^{2}$, Akira Chikamoto $^{1}$, Toru Beppu ${ }^{1}$, \\ Michio Kawasuji ${ }^{2}$, Hideo Baba $^{1^{*}}$ \\ ${ }^{1}$ Department of Gastroenterological Surgery, Kumamoto University Graduate School of Medical Sciences, Kumamoto, Japan; \\ *Corresponding Author: hdobaba@kumamoto-u.ac.jp \\ ${ }^{2}$ Department of Cardiovascular Surgery, Kumamoto University Graduate School of Medical Sciences, Kumamoto, Japan
}

Received 7 June 2013; revised 8 July 2013; accepted 20 July 2013

Copyright (C) 2013 Yashiro Motooka et al. This is an open access article distributed under the Creative Commons Attribution License, which permits unrestricted use, distribution, and reproduction in any medium, provided the original work is properly cited.

\begin{abstract}
Leiomyosarcoma of the inferior vena cava (IVC) is a rare tumor, and it needs complete surgical resection for cure. In addition, the reconstruction of IVC is necessary in many cases. Herein, we indicate the case of a 57-year-old female with leiomyosarcoma in segment I of the IVC, which grew deep into vascular lumen. She underwent complete en bloc resection of the tumor and IVC reconstruction by an artificial pericardium patch.
\end{abstract}

Keywords: Leiomyosarcoma; Inferior Vena Cava; Reconstruct

\section{INTRODUCTION}

Leiomyosarcoma is a rare malignant tumor of smooth muscle cell, and rarely develops from inferior vena cava (IVC) with intraluminal and/or extraluminal growth [1-6]. Because the long-term survival is not favorable (5-year survival 38\%, 10-year survival 14\%) [7], curative en bloc resection with IVC and reconstruction of that is important to cure. The location of leiomyosarcoma of the IVC is divided into three levels: segment I, lower level (IVC below the renal veins); segment II, middle level (renal veins to hepatic veins, most frequently affected); and segment III, upper level (entry of hepatic veins to the right atrium) [8].

In this report, we indicate the case of a 57-year-old female with leiomyosarcoma in segment I of the IVC. She underwent complete en bloc resection of the tumor and IVC reconstruction.

\section{CASE REPORT}

A 57-year-old female with no complaint received health screening abdominal ultrasound, which found retroperi- toneal tumor. Enhanced computed tomography (CT) scan revealed a tumor (diameter: $2.5 \mathrm{~cm}$ ) on the right side of IVC with growth into the IVC (Figures 1(A) and (B)). Maximum standardized uptake value (SUV max) of the tumor in positron emission tomography (PET)-CT was $4.4 \rightarrow 5.3$, and no distant metastasis was found.

Therefore, we planned a surgical resection with the patient's informed consent. Laparotomy showed that $30 \mathrm{~mm}$ tumor existed on the right side wall of IVC below the right renal vein junction (segment I), invading IVC widely (Figure 2(A)). An incision was made onto the clamped IVC, and it showed that the tumor protruded into the cavity of IVC. The tumor was resected en-block with IVC. Deficient hole of IVC was $2.5 \times 2.3 \mathrm{~cm}$ (Figure 2(B)), and reconstructed by GORE-TEX ${ }^{\circledR}$ Cardiovascular Patch (W. L. Gore \& Associates, Inc. Flagstaff,

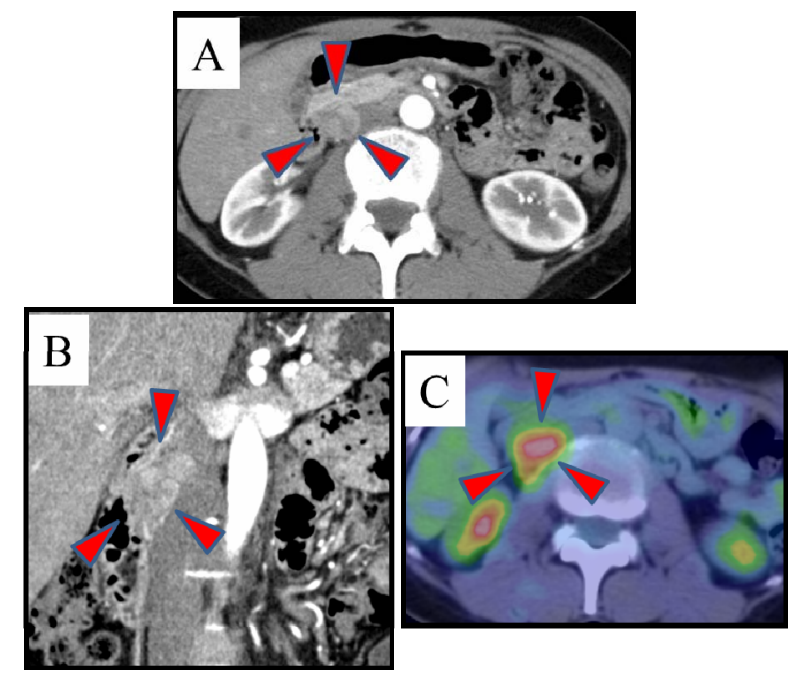

Figure 1. Preoperative findings. Enhanced CT revealed a tumor (arrowheads) on the right side of IVC with growth into the IVC ((A), (B)). SUV max of the tumor (arrowheads) in positron emission tomography (PET)-CT was $4.4 \rightarrow 5.3(\mathrm{C})$. 
AZ) with 6-0 polypropylene non-absorbable monofilament suturing (Figure 2(C)). Macroscopically, the tumor was elastic hard and the size was $5 \times 2.5 \times 2 \mathrm{~cm}$ (Figure 3(A)). Postoperative pathological diagnosis was leiomyosarcoma (Figure 3(B)). No sign of recurrence was ob-

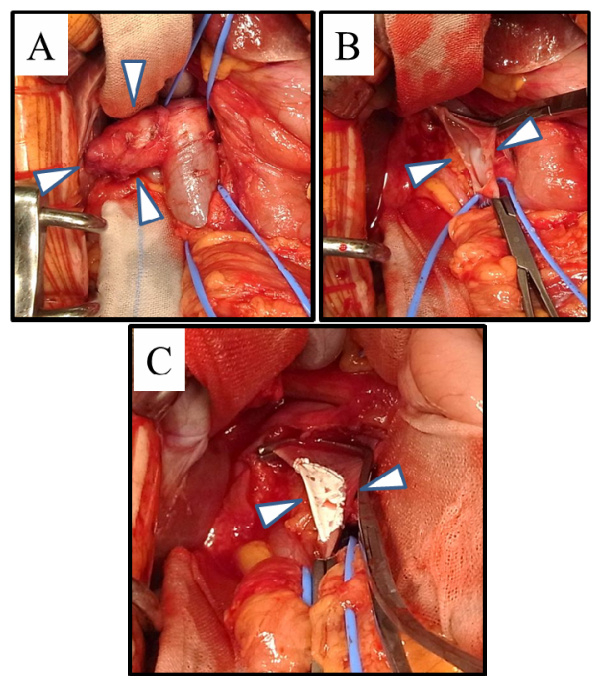

Figure 2. Surgical findings. Laparotomy showed that a tumor (arrowheads) existed on the right side wall of IVC below the right renal vein junction (segment I), invading IVC widely (A). Deficient hole (arrowheads) of IVC after en-block resection of the tumor was 2.5 $\times 2.3 \mathrm{~cm}(\mathrm{~B})$. Reconstructed by GORE-TEX ${ }^{\mathbb{B}}$ Cardiovascular Patch (arrowheads) was performed (C).

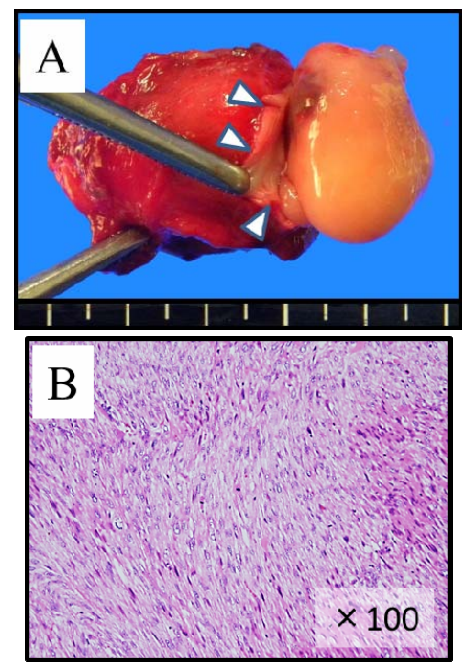

Figure 3. Pathological findings. Macroscopically, the tumor was elastic hard and the size was $5 \times 2.5 \times 2 \mathrm{~cm}$ (A) (arrowheads; resected IVC). HE staining showed cellular eosinophilic spindle cell tumor with nuclear atypia and mitosis, then pathological diagnosis was leiomyosarcoma (B). served and the patency of IVC was retained completely 10 months after the operation.

\section{DISCUSSION}

Aggressive curative en bloc surgical treatment to achieve extirpation of primary caval neoplasms or large abdominal tumors with caval involvement may prolong survival in selected patients [1-5]. Whereas a complete resection of the IVC is necessary in most cases, management of IVC after tumor resection is still controversial [8]. IVC reconstruction is performed by such as primary ligation, cavoplasty or graft replacement [9].

Kieffer et al. indicated that simple ligation is possible after complete or subtotal resection of the infra-renal IVC (segment I) with the assumption that slow tumor growth allows sufficient collaterals developing [8]. However, in cases without enough sufficient collateral growing, simple ligation can make low limb edema with significant functional impairment [9]. Thanks to advances in surgical techniques for venous reconstruction, prosthetic replacement of the IVC is now feasible whenever considered necessary [8].

In this case, leiomyosarcoma invaded the IVC widely. However, the IVC was not obstructed, and then collaterals were not developed. Aggressive curative en bloc surgical resection with IVC and reconstruction with an artificial patch were performed successfully and showed the successful long-term outcome without tumor recurrence. The patency of IVC has been kept, without any thrombosis.

\section{REFERENCES}

[1] Cho, S.W., Marsh, J.W., Geller, D.A., Holtzman, M., Zeh, H., Bartlett, D.L., et al. (2008) Surgical management of leiomyosarcoma of the inferior vena cava. Journal of Gastrointestinal Surgery, 12, 2141-2148. doi:10.1007/s11605-008-0700-y

[2] Wang, Q., Jiang, J., Wang, C., Lian, G., Jin, M.S. and Cao, X. (2012) Leiomyosarcoma of the inferior vena cava level II involvement: Curative resection and reconstruction of renal veins. World Journal of Surgical Oncology, 10, 120. doi:10.1186/1477-7819-10-120

[3] Alexander, A., Rehders, A., Raffel, A., Poremba, C., Knoefel, W.T. and Eisenberger, C.F. (2009) Leiomyosarcoma of the inferior vena cava: Radical surgery and vascular reconstruction. World Journal of Surgical Oncology, 7, 56. doi:10.1186/1477-7819-7-56

[4] Stauffer, J.A., Fakhre, G.P., Dougherty, M.K., Nakhleh, R.E., Maples, W.J. and Nguyen, J.H. (2009) Pancreatic and multiorgan resection with inferior vena cava reconstruction for retroperitoneal leiomyosarcoma. World Journal of Surgical Oncology, 7, 3. doi:10.1186/1477-7819-7-3

[5] Kyriazi, M.A., Stafyla, V.K., Chatzinikolaou, I., Koureas, 
A., Chatziioannou, A., Kondi-Paphiti, A., et al. (2010) Surgical challenges in the treatment of leiomyosarcoma of the inferior vena cava: Analysis of two cases and brief review of the literature. Annals of Vascular Surgery, 24, 826.e13-826.e17.

[6] Dew, J., Hansen, K., Hammon, J., McCoy, T., Levine, E.A. and Shen, P. (2005) Leiomyosarcoma of the inferior vena cava: Surgical management and clinical results. American Journal of Surgery, 71, 497-501.

[7] Mingoli, A., Cavallaro, A., Sapienza, P., Di Marzo, L., Feldhaus, R.J. and Cavallari, N. (1996) International registry of inferior vena cava leiomyosarcoma: Analysis of a world series on 218 patients. Anticancer Research, 16, 3201-3205.

[8] Kieffer, E., Alaoui, M., Piette, J.C., Cacoub, P. and Chiche, L. (2006) Leiomyosarcoma of the inferior vena cava: Experience in 22 cases. Annals of Surgery, 244, 289-295. doi:10.1097/01.sla.0000229964.71743.db

[9] Yoshidome, H., Takeuchi, D., Ito, H., Kimura, F., Shimizu, H., Ambiru, S., et al. (2005) Should the inferior vena cava be reconstructed after resection for malignant tumors? American Journal of Surgery, 189, 419-424. doi:10.1016/j.amjsurg.2005.01.010 